\title{
Monitoring System of Saturation Line Based on Mixed Programming
}

\author{
Pengrui Qiu, 2, a, Xiping Yuan, b, Shu Gan, c, Jianming Zhang, d, Min Yang ${ }^{1, \text { e }}$ \\ 1Kunming University of Science and Technology, Kunming 650093, China. \\ ${ }^{2}$ Kunming Metallurgy College, Kunming 650033, China.
}

a80420631@qq.com, bxpyuan@126.com, cshug@qq.com, djianmingz@qq.com, eminycc@qq.com

\begin{abstract}
Keywords: MATLAB, VC++, Saturation Line, Monitoring, Mixed Programming.
\end{abstract}
\begin{abstract}
After the impoundment of the reservoir, in the role of the water head, there will be seepage in the dam, which will produce the seepage of the dam, the dam cross section and intersection called saturation line. Saturation line is the lifeline of the safety of tailings dam, saturation line height is directly related to the dam stability and safety properties, when the saturation line depth is $0 \mathrm{~m}$, this will produce piping, which is the direct cause of the dam break Introduction. In water conservancy and hydropower, it is mainly to monitor the location and change of the line of the dam by calculating the height of the saturation line, determining the influence of the stability of the dam, in order to determine whether the dam is stable, to ensure the safe operation of the dam. Capacity, flow rate, precipitation can be intuitive monitoring of hydrological parameters Compared with above parameters monitoring saturation line is hidden, the monitoring and analysis is real-time and intuitive, automation and intelligent, so the system chooses mixed programming of MATLAB and VC ++ to achieve saturation line monitoring.
\end{abstract}

\section{Introduction}

Water conservancy and hydropower, after the impoundment of the reservoir, in the role of the water head, the dam will appear seepage, leakage in dam body, as gradual and stable seepage and it will produce stable water level in the dam body, the so-called saturation line, curve of the formation of the stable seepage face on the line of the dam body cross section. Calculation saturation line in water conservancy and hydropower is mainly to acquire its height, in order to determine the impact on the stability of the dam.

The mainly safety factors of reservoir dam includes infiltration line height, the dam body displacement, reservoir water level technical parameters, and the running state of the saturation line height can accurately the response of reservoir dam, playing dominant role in a number of reservoir dam safety factors, so real-time monitoring of saturation line height is effective direct means of monitoring reservoir dam running state. The research object of the saturation line of the reservoir at home and abroad mainly focuses tailings dam and it is not much. In our country, the role of the majority of reservoirs is to provide drinking water, agricultural irrigation and flood control, this paper for the large number of reservoirs, combined with the actual needs of the engineering to design the system of saturation line monitoring. The monitoring of the actual saturation line of the dam is an effective means for people to understand the operation state and safety condition of the reservoir dam. Saturation line monitoring system of reservoir dam is a part of the hydrological monitoring system, with the water conservancy information acquisition, processing, sharing, greatly enhance the degree of information and need to more convenient and intelligent saturation line monitoring system, improve information and ability of water conservancy project construction and management. Saturation line monitoring system of reservoir dam is for the real-time and accurate grasp of reservoir dam stability, once the monitoring to the stability of the reservoir dam discovery damage it will give a warning, suggesting the staff of reservoir dam reinforcement to ensure the safe operation of the reservoir dam, the reservoir can exert the benefit of project and the benefit of flood control. Saturation line monitoring system of reservoir dam to realize the monitoring level of data acquisition and transmission, data storage, data analysis and information release[1][2]. 


\section{System Design}

The upstream river seeps through the dyke to the lower reaches under gravity, forming a free surface that flows down gradually in the internal body calling phreatic surface, the line of intersection between the phreatic surface and the dike section is called the saturation line. The height and variation of the saturation line are closely related to the seepage safety of the dam. If the saturation line is higher than the design value, it will reduce the slope stability, and even cause the instability of landslide.

In order to protect the safety of the dam, to provide the first hand data, to monitor real-time water-height data that reservoir dam is penetrated by water, according to the design of saturation line it can know the height of the water level of the security level, compared with the point of the actual water level height and safe water level height, statistics and analysis the comparison results, at last the system can be a preliminary judgment of the stability of the reservoir dam.

Due to the actual monitoring points of saturation being far away from the reservoir in control room and reservoir working environment in the field is very bad, the use of cable power supply and communication leading to these lines affected by the rain and sunlight and other factors of corrosion. The stability of the line is greatly reduced, the construction is difficult, large investment and once the fault is not difficult to find and repair. Reservoirs always located in the remote area, the electromagnetic interference is small, the choice of wireless communication and wired communication can greatly improve the stability and accuracy of the communication, the system can adopt modular design, once a monitoring point acquisition and data transmission appears problems, staff just replaces the monitoring module to make the point rework so as to improve the monitoring system's stability, reliability, practicability and flexibility[3][4]. MATLAB Engine is the interface function provided by MATLAB. By using it, VC++ can call the interface function directly to fulfill the requirement.

In this paper, the monitoring system through GPRS and Zigbee and other means of communication reads data from the sensor from the various points, to send data to the host computer. Data acquisition module is mainly constituted by STM32 that uses it reading the data of hydrological sensor transmitter. Then the data of hydrological sensor will be sent to the GPRS DTU and ZigBee DTU through RS-485 protocol after A / D conversion. Considering the operation cost, the communication between monitoring stations and host computer uses GPRS and ZigBee protocol, short- distance communication using ZigBee, long-distance communication using GPRS, that the two communications complement each other.

In order to distinguish different monitoring points, the system chooses Modbus RTU protocol. The protocol is divided into master station and slave station. Modbus Network includes only one master station and 256 slave stations. Master station sends data command to read or write register of the slave which the command includes the slave address, function code, data. The master station can only poll a slave station once time, so it needs to send polling data command which is issued to all the slaves in the Modbus network. If the slave has received the data command to check the data to judge whether the command is sent to itself. If the result is not, the slave will not make the corresponding response. The way ensures the data transfer on purpose and it will avoid the data chaos at the same time in the Modbus Network. When the slave station receiving command from master station, it analyses the command to make a decision whether to response to master station. To improve the data transmission safety, the slave station will make the data to execute CRC check comparing to the received CRC value.

VC and MATLAB are used as the host computer, VC through MATLAB Engine interface calls MATLAB program to processing data, according to the model constructed in advance to obtain the reservoir operation state, determine whether to alarm the information, namely if actual saturation line is higher than the design of saturation line, automatic alarm messages and inform the personnel on duty[5][6]. Through calling the engOpen(), open the MATLAB Engine. VC++ makes the data which come from hydrological sensor transmitter send to the MATLAB via calling the engEvalString(). The working diagram of the system is shown in figure.1. 


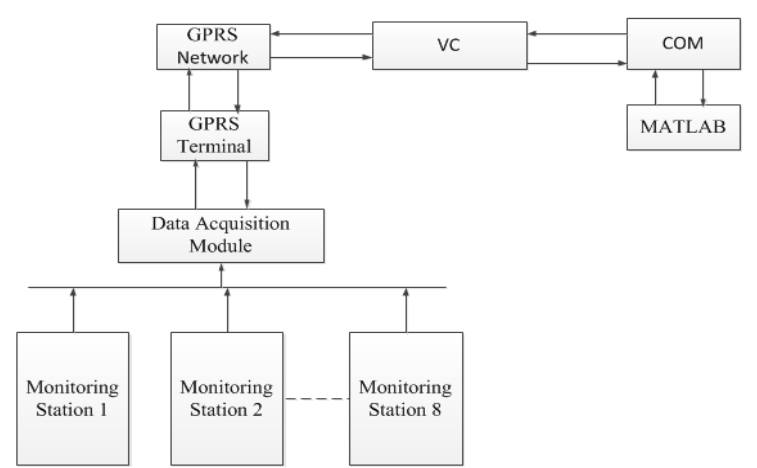

Fig. 1 Working diagram of the system

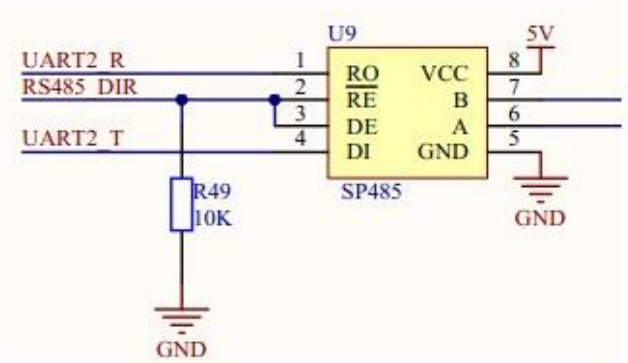

Fig. 2 Saturation line comparing

\section{Hardware Design}

Data acquisition module is mainly constituted by STM32F103ZET6, and it belongs to the 32 bit Cortex-M3of ARM. It can achieve Maximum 72MHz operating frequency and has the characteristics of single cycle multiplication and hardware division. The data obtaining from each monitoring point is analog and must be converted by A/D to be uploaded to the host computer. The accuracy of the data depends on the number of bits A/D and STM32 has two of 12 bit A/D converters with 1US conversion time (up to 16 input channels) to make sure accuracy. The communication between STM32 and sensor uses RS-485 protocol.

The maximum transmission distance of the RS-485 protocol is $4000 \mathrm{feet}$, actually up to 3000 meters comparing with RS-232 protocol that only is allowed to connect 1 transceivers on the bus, namely single station capacity. The RS-485 protocol is allowed to connect up to 128 transceivers on the bus. That is multi station capability, so that users can easily establish a device network using a single RS-485 protocol. The schematic of STM32 and MAX485 is shown in Figure2.

\section{Software Design}

VC support object-oriented program design, process oriented design and automatic generation of applications, strong ability of graphical interface editing, the software development efficiency being high; but the ability of analysis and the processing data is weak, not suitable for large data processing and analysis. MATLAB provides a variety of professional toolbox, with powerful numerical calculation, data processing, graphical analysis and symbolic computation, improving the efficiency of engineering calculation, so it can help the user to solve the major problems in the field of complex[7][8].

VC and MATLAB mixed programming have a variety of ways, the system chooses the way based on MATLAB Engine technology. MATLAB prepared to convert the program into COM components, $\mathrm{VC}$ in the application to follow the MATLAB Engine interface standard to call it. This method is simple and universal, and can use any function of MATLAB [9]. In water conservancy and hydropower, it is mainly by calculating the height of the saturation line to monitored position and the change of the saturation line of the dam to determine the impact on the stability of the dam. In the analysis of the saturation line of the host computer, it is to compare the actual saturation line equation and the design saturation line equation. Because the system can only get some discrete points, it must do the curve fitting. Before fitting the curve, it is necessary to know the curve form of the reservoir.

$$
\begin{aligned}
& h(\lambda)=0.1091 \lambda^{4}-0.7501 \lambda^{3}+1.9283 \lambda^{2}-2.2319 \lambda+1 \\
& \mu \_ \text {water supply } \\
& h_{m-\text { water head height }} \\
& t_{-} \text {time } \\
& h \_ \text {height of saturation line } \\
& x \_ \text {dam distance }
\end{aligned}
$$


The result is obtained by the fitted polynomial subtracting the design infiltration line function polynomial. If the result is less than 0 , the reservoir saturation line in a safe state, conversely in a dangerous state and alarming. Comparison of curve fitting with design saturation line is shown in Figure3.

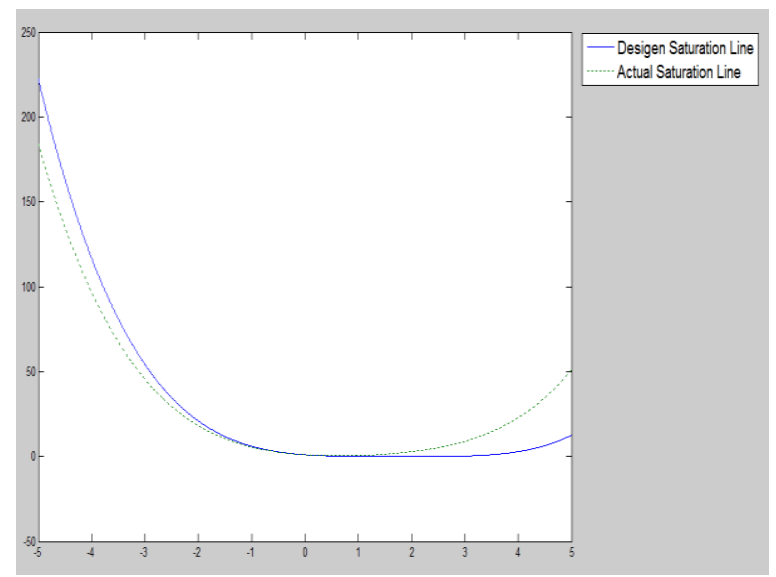

Fig. 3 Saturation line comparing

\section{Conclusion}

The monitoring system timely accurate get each monitoring point data and curve fitting, to quickly determine the relationship between the design value and the monitor value, truly realize the reservoir saturation line intelligent monitoring.

\section{References}

[1]. Ping Ai. Research and Practice on information evaluation of water resources in China[M]. Changjiang Publishing, 2011:1-5.

[2]. Niv Goldenberg, Avishai Wool. Accurate Modeling of Modbus/TCP for Intrusion Detection in SCADA Systems[J].International Journal of Critical Infrastructure Protection, 6(2013) : 63-75.

[3]. Baimin Zhang. Application of Computer Monitoring system of Sluice Gate in the south of Kong[J]. Hydropower Automation and Dam Monitoring, 34(2010):82-84.

[4]. Xinyou Zhang. THE RESEARCH AND APPLICATION OF THE AUTOMATIC MONITORING SYSTEM OF WATER REGIMEN BASED ON GPRS[D]. Zhejiang University of Technology,2009,36-46.

[5]. Derwin Suhartono.Developing Controller Area Network Management Application Based on Modbus in Multi Generator Set Controller through Local Network and Internet[J]. Procedia Engineering, 2012(50): 426-435.

[6]. Yu Lin. Automatic Control System of Hydrological Data Acquisition of Reservoir Gate[D]. Kunming University of Science and Technology, 2014, 30-50.

[7]. Maria Auxiliadora Muanis Persechini.Centralized and Distributed Control Architectures under Foundation Fieldbus Network[J]. ISA Transactions, 52(2013):149-161.

[8]. Sujuan Tang, Jianfeng Mao. Application of automatic water regime measuring system via GPRS network at Wanyao reservoir[J]. Large Dam \& Safety, 2(2011):114-116.

[9]. Yufeng Ding.Proficient in MATLAB Mixed Programming[M]. Publishing House of Electronics Industry, 2012, 3-88. 